Pacific Journal of Mathematics

NONEXPANSIVE PROJECTIONS ON SUBSETS OF BANACH 


\section{NONEXPANSIVE PROJECTIONS ON SUBSETS OF BANACH SPACES}

Ronald E. Bruck, JR.

If $C$ is a convex subset of a Banach space $E$, a projection is a retraction $r$ of $C$ onto a subset $F$ which for each $x \in C$ maps each point of the ray $\{r(x)+t(x-r(x)): t \geqq 0\} \cap C$ onto the same point $r(x)$. A retraction $r$ is said to be orthogonal if for each $x, x-r(x)$ is normal to $F$ in a sense related to that of R. C. James. This paper establishes three main results. First, a nonexpansive projection is necessarily an orthogonal retraction; if $E$ is smooth, the converse is also true. Second, if $E$ is smooth then there can exist at most one nonexpansive projection of $C$ onto a given subset $F$. Third, if $E$ is uniformly smooth and there exists a nonexpansive retraction of $C$ onto $F$, then there exists a nonexpansive projection of $C$ onto $F$. The proximity mapping is a nonexpansive projection in a Hilbert space, but not in a general Banach space.

We shall adopt the following conventions throughout this paper: $E$ always denotes a real Banach space, $E^{*}$ its dual space, $C$ a nonempty closed convex subset of $E$, and $F$ a nonempty closed subset of $C$. We do not assume that $F$ is convex. A mapping $f: C \rightarrow C$ is said to be nonexpansive if $\|f(x)-f(y)\| \leqq\|x-y\|$ for all $x, y \in C$. $F$ is said to be a nonexpansive retract of $C$ if there exists a retraction of $C$ onto $F$ which is a nonexpansive mapping.

Nonexpansive retracts are of interest because they generalize two results, one linear in reflexive Banach spaces, one nonlinear in Hilbert space.

First, if $E$ is reflexive and $L: E \rightarrow E$ is linear with $\|L\| \leqq 1$, then the mean ergodic theorem implies that the means $L_{n}=n^{-1} \sum_{j=1}^{n} L^{j}$ converge pointwise to a projection $P$ of $E$ onto $\operatorname{Ker}(I-L)$, and $\|P\| \leqq 1$. The kernel of $I-L$ is the fixed-point set of $L$.

Second, if $E$ is a Hilbert space and $T: C \rightarrow C$ is nonexpansive, then $F(T)$, the fixed-point set of $T$, is a closed convex subset of $C$. If $F(T)$ is nonempty, the proximity mapping $P: C \rightarrow F(T)$ defined by $P x=$ the point of $F(T)$ which is closest to $x$, is known to be nonexpansive. In fact, it satisfies the stronger inequality

$$
\|P x-P y\|^{2} \leqq(x-y, P x-P y)
$$

for all $x, y$ in $C$ (Browder [3], Prop. 2).

In both of these examples the fixed-point set of a nonexpansive mapping: $C \rightarrow C$ is shown to be a nonexpansive retract of $C$, and a nonexpansive retraction is constructed which has a pseudolinearity 
property, namely, it projects $C$ onto the fixed-point set along rays. In Bruck [4] it was shown that if $E$ is a reflexive, strictly convex Banach space and $T: C \rightarrow C$ is nonexpansive, then $F(T)$ is a nonexpansive retract of $C$. In this paper we show that under certain additional hypotheses of smoothness, this retraction can be taken to be a projection (in the sense of the abstract). Such a projection is unique and maps $C$ onto $F(T)$ along rays which are normal to $F(T)$.

Although the proximity map of $C$ onto a closed convex subset $F$ can be defined in any strictly convex, reflexive space, and when defined is a projection, it is not, in general, nonexpansive (de Figueiredo and Karlovitz [6]), nor does it project $C$ onto $F$ along normals. In fact, if $F$ is bounded, convex, and smooth, while $E$ is finite-dimensional, it was shown in [5] that there does not exist any nonexpansive retraction of $E$ onto $F$ unless $E$ is a Hilbert space or $\operatorname{dim} E=2$. Thus the class of mappings we study, the nonexpansive projections, is new and distinct from the class of proximity mappings.

Another motivation for the study of nonexpansive retracts is that they are precisely the subsets $F$ of $C$ for which the following extension problem can be solved for each choice of $T$ and $M$ :

Given a Lipschitzian map $T$ of $F$ into a metric space $M$, to (LEP) extend $T$ to a map $T^{*}: C \rightarrow M$ without increasing the Lipschitz constant of $T$.

If $r$ is a nonexpansive retraction of $C$ onto $F$, (LEP) can be solved by letting $T^{\#}=T \circ r$; while if (LEP) can be solved on $F$ for each $T$ and $M$, then it can be solved for $M=F$ and $T=$ identity, in which case $T^{\#}$ is a nonexpansive retraction of $C$ onto $F$.

In particular, if the nonexpansive retraction $r$ can be taken to be a projection, the extension of $T$ to $T^{\#}$ is greatly facilitated since $T^{\ddagger}$ is constant along certain rays emanating from $F$ and need be calculated only in some neighborhood of $F$.

1. Orthogonality and the semi-inner-product. The definition of orthogonality we use is that of Birkhoff [1] and James [8]: $x$ is said to be orthogonal to $y$ if

$$
\|x+t y\| \geqq\|x\|
$$

for all $t \in R^{1}$. We extend this definition as follows:

Definition $1 . x$ is said to be acute to $y$ if (2) holds for all $t \geqq 0$. 
Definition 2. A retraction $r$ of $C$ onto $F$ is orthogonal if for each $p \in C$ and $y \in F, r(p)-y$ is acute to $p-1 r(p)$ :

$$
\|(1-t) r(p)+t p-y\| \geqq\|r(p)-y\| \text { for all } t \geqq 0 \text {. }
$$

This definition is suggested by the following observation:

Proposition 1. Suppose $r$ is an orthogonal retraction of $E$ onto a convex subset $F$. If for some $x_{0} \in E, F$ has a unique supporting hyperplane $H+r\left(x_{0}\right)$ at its boundary point $r\left(x_{0}\right)$, then each $u \in H$ is orthogonal to $r\left(x_{0}\right)-x_{0}$.

Proof. By hypothesis, for each $y \in F$,

$$
\left\|(1-t) r\left(x_{0}\right)+t x_{0}-y\right\| \geqq\left\|r\left(x_{0}\right)-y\right\| \text { for all } t \geqq 0 .
$$

If $K$ denotes the cone with vertex $r\left(x_{0}\right)$ generated by $F$,

$$
K=\bigcup_{\lambda>0}(1-\lambda) r\left(x_{0}\right)+\lambda F,
$$

then (4) holds for $y \in K$ as well. For, if $\lambda>0, y_{0} \in F$, and $y=$ $(1-\lambda) r\left(x_{0}\right)+\lambda y_{0}$, then

$$
\begin{aligned}
& \left\|(1-t) r\left(x_{0}\right)+t x_{0}-y\right\|=\lambda\left\|\left(1-\frac{t}{\lambda}\right) r\left(x_{0}\right)+\frac{t}{\lambda} x_{0}-y_{0}\right\| \\
& \quad \geqq \lambda\left\|r\left(x_{0}\right)-y_{0}\right\|=\left\|r\left(x_{0}\right)-y\right\|
\end{aligned}
$$

since (4) holds for $t / \lambda$ as well as for $t$.

Clearly (4) also holds for all $y \in \bar{K}$, the closure of $K$. Since $F$ has a unique supporting hyperplane $H+r\left(x_{0}\right)$ at $r\left(x_{0}\right), \bar{K}$ is the closed half-space with boundary $H+r\left(x_{0}\right)$, which contains $F$. Therefore, $H+r\left(x_{0}\right) \subset \bar{K}$. For any $u \in H$ we have also $-u \in H$; since $H+r\left(x_{0}\right) \subset$ $\bar{K}$, we may take $y= \pm u+r\left(x_{0}\right)$ in (4), obtaining

$$
\left\|u \pm t\left(r\left(x_{0}\right)-x_{0}\right)\right\| \geqq\|u\|
$$

for all $t \geqq 0$. Therefore, each $u \in H$ is orthogonal to $r\left(x_{0}\right)-x_{0}$.

Let $S=\{u \in E:\|u\|=1\}$.

Definition 3. The Banach space $E$ is smooth if for each $(x, y) \in$ $S \times S$ the limit

$$
\lim _{t \rightarrow 0} t^{-1}(\|x+t y\|-\|x\|)
$$

exists. $E$ is uniformly smooth if the limit (5) is uniform for $(x, y) \in$ $S \times S$.

Equivalently, $E$ is smooth iff at each point of $S$ there is only 
one supporting hyperplane to the unit ball; $E$ is uniformly smooth iff $E^{*}$ is uniformly convex (Smulian [11]).

DEFINITION 4. If $E$ is smooth, the semi-inner-product $[\cdot, \cdot \cdot]$ on $E$ is the Gateaux differential of $1 / 2\|\cdot\|^{2}$ (with arguments reversed):

$$
[y, x]=\lim _{t \rightarrow 0} t^{-1}\left(\frac{1}{2}\|x+t y\|^{2}-\frac{1}{2}\|x\|^{2}\right) .
$$

This limit exists for each $x, y \in E$ by virtue of the existence of (5) for each $x, y \in S$.

PRoposition 2. If $E$ is smooth, the semi-inner-product satisfies the following:

(a) $[\cdot, x]$ is linear for each $x \in E$;

(b) $[x, x]=\|x\|^{2}$;

(c) $|[y, x]| \leqq\|y\| \cdot\|x\|$;

(d) $[y, \lambda x]=\lambda[y, x]$

(e) $[\cdot, \cdot]$ is continuous on $E \times E$.

If $E$ is uniformly smooth, the semi-inner-product is uniformly continuous on bounded subsets of $E \times E$.

REMARK. Lumer [10] defined the semi-inner-product to be any function $[\cdot, \cdot]: E \times E \rightarrow R^{1}$ which satisfies (a)-(c) of Proposition 2. For our later purposes the continuity condition (e) is essential. However, it follows from a result of Giles ([7], p. 439) that if $[\cdot, \cdot]$ satisfies (a)-(c) and (e), then $E$ is smooth and $[y, x]$ is given by the limit (6). Hence there is no point in our using the more general definition.

Proof. (b), (c), and (d) follow directly from the definition of $[y, x]$ as the limit (6). (a) and (e) are true of the Gateaux differential of any continuous convex functional $f$ for which $\lim _{t \rightarrow 0} t^{-1}(f(x+t y)-f(x))$ exists for each $x, y \in E$. Finally, if $E$ is uniformly smooth, then the limit (6) is uniform on bounded sets of $x, y$ and $1 / 2\|\cdot\|^{2}$ is uniformly continuous on bounded sets. If $f$ is any functional (convex or not!) which is uniformly continuous on bounded sets and for which $g(x, y)=$ $\lim _{t \rightarrow 0} t^{-1}(f(x+t y)-f(x))$ exists uniformly on bounded sets, it is easy to see that $g$ is uniformly continuous on bounded sets.

Proposition 3. Suppose $E$ is smooth. Then $x$ is orthogonal to $y$ iff $[y, x]=0 ; x$ is acute to $y$ iff $[y, x] \geqq 0$.

Proof. We will prove the equivalence for $x$ acute to $y$, the other part being similar. 
Let $f(t)=1 / 2\|x+t y\|^{2}$. Then $f$ is differentiable and $f^{\prime}(t)=$ $[y, x+t y]$.

By definition, $x$ is acute to $y$ iff $f(t) \geqq f(0)$ for all $t \geqq 0$. Since $f$ is convex, this is so iff $f^{\prime}(0) \geqq 0$, i.e., $[y, x] \geqq 0$.

2. Approximating the nonexpansive retraction. We will prove our main results with the aid of an approximation scheme of F. E. Browder [2].

Definition 5. If $p \in E$ and $F \neq \varnothing$, the approximation region between $p$ and $F$ is the set

$A(p ; F)=\{u \in E: u-y$ is acute to $p-u$ for all $y \in F\}$.

Equivalently, $A(p ; F)$ is the set of $u$ which satisfy

$$
\|(1-t) u+t p-y\| \geqq\|u-y\|
$$

for all $t \geqq 0$ and $y \in F$. If $E$ is smooth, by Prop. 3 and Prop. 2(d)

$$
A(p ; F)=\{u \in E:[u-p, u-y] \leqq 0 \text { for all } y \in F\} .
$$

Referring to Def. 2, we also have the following characterization of orthogonal retractions:

(8) $r$ is an orthogonal retraction of $C$ onto $F$ iff $r(p) \in F \cap A(p ; F)$ for each $p \in C$.

Lemma 1. The approximation region $A(p ; F)$ is always closed and bounded. If $E$ is smooth then $F \cap A(p ; F)$ can contain at most one point.

Proof. Suppose $\left\{u_{n}\right\}$ is a sequence in $A(p ; F)$ and $u_{n} \rightarrow u$. Then for each $t \geqq 0$ and $y \in F$,

$$
\left\|(1-t) u_{n}+t p-y\right\| \geqq\left\|u_{n}-y\right\| \text {. }
$$

Letting $n \rightarrow \infty$, we find

$$
\|(1-t) u+t p-y\| \geqq\|u-y\|,
$$

so $u \in A(p ; F)$. Therefore, the approximation region is closed.

Next, suppose $u \in A(p ; F)$. Then (9) holds for $t=1$ in particular, so $\|p-y\| \geqq\|u-y\|$. Thus $A(p ; F)$ is a subset of the ball of radius $\|p-y\|$, centered at $y$. The approximation region is therefore bounded. (Recall that $F$ is assumed to be nonempty.)

Finally, suppose $E$ is smooth and $u_{1}, u_{2} \in F \cap A(p ; F)$. By the equivalent formulation (7) of $A(p ; F)$ in the smooth case, 


$$
\left[u_{i}-p, u_{i}-y\right] \leqq 0
$$

for $i=1,2$ and $y \in F$. Since $u_{1}, u_{2} \in F$ we may take $i=1, y=u_{2}$ in (10), obtaining

$$
\left[u_{1}-p, u_{1}-u_{2}\right] \leqq 0,
$$

then $i=2, y=u_{1}$, obtaining

$$
\left[u_{2}-p, u_{2}-u_{1}\right] \leqq 0 .
$$

By Prop. 2, $\left[u_{2}-p, u_{2}-u_{1}\right]=\left[p-u_{2}, u_{1}-u_{2}\right]$; thus

$$
\left[p-u_{2}, u_{1}-u_{2}\right] \leqq 0 \text {. }
$$

Adding (11) and (12),

$$
\left[u_{1}-u_{2}, u_{1}-u_{2}\right]=\left[u_{1}-p+p-u_{2}, u_{1}-u_{2}\right] \leqq 0 .
$$

But $\left[u_{1}-u_{2}, u_{1}-u_{2}\right]=\left\|u_{1}-u_{2}\right\|^{2}$, therefore $u_{1}=u_{2}$.

Lemma 2. Suppose $E$ is uniformly smooth and $\left\{x_{n}\right\}$ is a sequence in $A(p ; F)$ such that $\lim _{n} \operatorname{dis}\left(x_{n}, F\right)=0$. Then $F \cap A(p ; F) \neq \varnothing$ and $\left\{x_{n}\right\}$ converges to the unique point in $F \cap A(p ; F)$.

Proof. Choose a sequence $\left\{y_{n}\right\}$ in $F$ so that $x_{n}-y_{n} \rightarrow 0$. By Lemma $1, A(p ; F)$ is bounded, so $\left\{x_{n}\right\}$ and $\left\{y_{n}\right\}$ are bounded.

By the uniform continuity of the semi-inner-product on bounded sets (Prop. 2),

$$
\lim _{n}\left(\left[y_{n}-p, y_{n}-y_{m}\right]-\left[x_{n}-p, x_{n}-y_{m}\right]\right)=0
$$

uniformly in $m$. But $\left[x_{n}-p, x_{n}-y_{m}\right] \leqq 0$ by (7), hence there exists a sequence $t_{n} \rightarrow 0+$ such that

$$
\left[y_{n}-p, y_{n}-y_{m}\right] \leqq t_{n} \text { for all } m, n \text {. }
$$

By symmetry,

$$
\left[y_{m}-p, y_{m}-y_{n}\right] \leqq t_{m} \quad \text { for all } m, n .
$$

Adding (13) and (14),

$$
\left\|y_{n}-y_{m}\right\|^{2}=\left[y_{n}-p+p-y_{m}, y_{n}-y_{m}\right] \leqq t_{n}+t_{m} .
$$

$\left\{y_{n}\right\}$ is therefore a Cauchy sequence in $F,\left\{x_{n}\right\}$ is a Cauchy sequence in $A(p ; F)$, and both sequences converge to the same limit $z$ since $x_{n}-y_{n} \rightarrow 0$. But since $F$ and $A(p ; F)$ are both closed, the limit $z$ belongs to $F \cap A(p ; F)$. 
Definition 6. A mapping $T: C \rightarrow E$ is firmly nonexpansive if for all $x, y \in C$ and $t \geqq 0$ there holds the inequality

$$
\|(1-t)(T x-T y)+t(x-y)\| \geqq\|T x-T y\| \cdot
$$

By taking $t=1$ we see that a firmly nonexpansive mapping is nonexpansive.

REMARK. Definition 6 requires that $T x-T y$ be acute to $(x-y)-$ $(T x-T y)$ for all $x, y \in C$. If $E$ is smooth, this implies

$$
[x-y-(T x-T y), T x-T y] \geqq 0
$$

by Prop. 3, so

$$
\|T x-T y\|^{2} \leqq[x-y, T x-T y] .
$$

Our terminology is therefore consistent with that of Browder [2], where, if $E$ is a Hilbert space, $T: C \rightarrow E$ is called firmly contractive if $\|T x-T y\|^{2} \leqq(x-y, T x-T y)$ for all $x, y \in C$ (see also (1)).

Our basic approximation result assures the existence of a large family of firmly nonexpansive mappings:

Lemma 3. Suppose $T: C \rightarrow C$ is nonexpansive and $F=\{y: T y=y\} \neq$ $\varnothing$. Then for each $\lambda \in(0,1)$ there is exactly one mapping $x_{\lambda}: C \rightarrow C$ with the property that $x_{\lambda}=\lambda T \circ x_{2}+(1-\lambda) I$, where $I=i d e n t i t y$. $x_{\lambda}$ and $T$ have the same fixed-point set, $x_{\lambda}$ is firmly nonexpansive, and $x_{\lambda}(p) \in A(p ; F)$ for each $p \in C$.

Proof. Since $0<\lambda<1$ and $T$ maps the convex set $C$ into itself, for fixed $p \in C$ the mapping $\lambda T+(1-\lambda) p$ is a $\lambda$-contraction of $C$ into $C$. By the Banach Contraction Principle, it has a unique fixed point. If $x_{\lambda}(p)$ denotes this fixed point, then $x_{\lambda}: C \rightarrow C$ has been defined so as to satisfy $x_{\lambda}=\lambda T \circ x_{\lambda}+(1-\lambda) I$; and by the uniqueness of the fixed point, there is only one such mapping $x_{\lambda}$.

If $x_{\lambda}(p)=p$, then $p=\lambda T(p)+(1-\lambda) p$, so $T(p)=p$. Conversely, if $T(p)=p$, then $p$ is a solution $z$ of $z=\lambda T z+(1-\lambda) p$; since $x_{\lambda}(p)$ is the only such solution, $x_{\lambda}(p)=p$. Therefore, $x_{\lambda}$ and $T$ have the same fixed-point set.

We see that $x_{\lambda}$ is firmly nonexpansive as follows: let $p, q \in C$, $u=x_{\lambda}(p), v=x_{\lambda}(q)$, so

$$
u-v=\lambda(T u-T v)+(1-\lambda)(p-q) .
$$

Let $w(s)=(1-s)(T u-T v)+s(p-q)$. Then $w(0)=T u-T v, w(1)=$ $p-q$, and, from (15), $w(1-\lambda)=u-v$. Since $0<1-\lambda<1, u-v$ lies on the line segment joining $T u-T v$ and $p-q$. Therefore, for 
any $t \geqq 0$ the three points $T u-T v, u-v$, and $(1-t)(u-v)+t(p-q)$ (in that order) are collinear. Since $\|T u-T v\| \leqq\|u-v\|$ it follows that

$$
\|(1-t)(u-v)+t(p-q)\| \geqq\|u-v\| \text { for all } t \geqq 0 .
$$

Substituting $u=x_{\lambda}(p)$ and $v=x_{\lambda}(q)$,

$$
\begin{array}{r}
\left\|(1-t)\left(x_{\lambda}(p)-x_{\lambda}(q)\right)+t(p-q)\right\| \geqq\left\|x_{\lambda}(p)-x_{\lambda}(q)\right\| \\
\text { for all } t \geqq 0,
\end{array}
$$

i.e., $x_{\lambda}$ is firmly nonexpansive.

Finally, $x_{\lambda}(p) \in A(p ; F)$. For any $y \in F$ we have $x_{\lambda}(y)=y$ since $x_{\lambda}$ and $T$ have the same fixed points. But $x_{\lambda}$ is firmly nonexpansive, so taking $q=y$ and noting that $x_{2}(y)=y$, (16) reduces to

$$
\left\|(1-t) x_{\lambda}(p)+t p-y\right\| \geqq\left\|x_{\lambda}(p)-y\right\|
$$

for all $t \geqq 0$ and $y$ in $F$, i.e., $x_{\lambda}(p) \in A(p ; F)$.

3. Projections and retractions. We are now in a position to prove our principal results.

DeFinition 7. A projection is a mapping $f$ which satisfies the following: whenever $p \in \operatorname{Dom} f, t \geqq 0$, and $p^{*}=f(p)+t(p-f(p))$ is in $\operatorname{Dom} f$, then $f\left(p^{*}\right)=f(p)$. Thus $f$ retracts its domain onto its range along rays. (In [4] such a mapping was called a ray retraction.)

THEOREM 1. Suppose $r$ is a retraction of $C$ onto $F$. Then each of the following conditions implies the next:

(a) $r$ is a nonexpansive projection;

(b) $r$ is firmly nonexpansive;

(c) $r$ is an orthogonal retraction.

If $E$ is smooth these conditions are equivalent and there can exist at most one nonexpansive projection of $C$ onto $F$.

Proof. $(\mathrm{a}) \Rightarrow(\mathrm{b})$. Suppose $r$ is a nonexpansive projection of $C$ onto $F$, and let the mappings $x_{\lambda}$ be defined as in Lemma 3 for $T=r$, i.e., $x_{\lambda}=\lambda r \circ x_{\lambda}+(1-\lambda) I$. Then (16) holds for all $t \geqq 0$.

Now $x_{\lambda}(p)=\lambda r\left(x_{\lambda}(p)\right)+(1-\lambda) p$, so $p$ lies on the ray from $r\left(x_{\lambda}(p)\right)$ which extends through $x_{\lambda}(p)$. Since $r$ is a projection, $r(p)=r\left(x_{\lambda}(p)\right)$, and hence $x_{\lambda}(p)=\lambda r(p)+(1-\lambda) p$. Therefore, $x_{\lambda}(p) \rightarrow r(p)$ and $x_{\lambda}(q) \rightarrow$ $r(q)$ strongly as $\lambda \rightarrow 1-$. Letting $\lambda \rightarrow 1-$ in (16), we obtain

$$
\|(1-t)(r(p)-r(q))+t(p-q)\| \geqq\|r(p)-r(q)\|
$$

for all $t \geqq 0$. That is, $r$ is firmly nonexpansive. 
(b) $\Rightarrow$ (c). If $r$ is a firmly nonexpansive retraction of $C$ onto $F$, then for $p$ in $C, q$ in $F$, (17) holds for all $t \geqq 0$, and $r(q)=q$. This reduces to (3) with $y=q$, so $r$ is orthogonal.

(c) $\Rightarrow$ (a) when $E$ is smooth. Whether or not $E$ is smooth, whenever $r$ is an orthogonal retraction of $C$ onto $F, p \in C, \lambda \geqq 0$, and $p^{*}=r(p)+\lambda(p-r(p))$, it follows that $r(p) \in A\left(p^{*} ; F\right) \cap F$. In fact, since $r$ is orthogonal $r(p)-y$ is acute to $p-r(p)$ for any $y$ in $F$, so for any $t \geqq 0$,

$$
\left\|r(p)-y+t\left(p^{*}-r(p)\right)\right\|=\|r(p)-y+\lambda t(p-r(p))\| \geqq\|r(p)-y\|
$$

since $p^{*}-r(p)=\lambda(p-r(p))$ and $\lambda t \geqq 0$. This shows that $r(p)-y$ is acute to $p^{*}-r(p)$, so $r(p) \in A\left(p^{*} ; F\right)$. Since $r(p) \in F$ also, we have $r(p) \in F \cap A\left(p^{*} ; F\right)$.

In particular, if $E$ is smooth and $p^{*} \in C$ then (8) and Lemma 1 imply that $F \cap A\left(p^{*} ; F\right)$ consists of the single point $r\left(p^{*}\right)$. Since $r(p)$ also belongs to this set, we must have $r\left(p^{*}\right)=r(p)$.

We have proven that if $E$ is smooth and $r$ is an orthogonal retraction, then $r$ is a projection; it remains to show that $r$ is nonexpansive. If $p, q \in C$ then $r(q) \in F$ so $r(p)-p$ must be acute to $r(q)-r(p)$, i.e.,

$$
[r(p)-p, r(p)-r(q)] \leqq 0
$$

By symmetry,

$$
[r(q)-q, r(q)-r(p)] \leqq 0 .
$$

Adding (18) and (19),

$$
[r(p)-r(q)-(p-q), r(p)-r(q)] \leqq 0,
$$

from which we conclude

$$
\|r(p)-r(q)\|^{2} \leqq[p-q, r(p)-r(q)] .
$$

But $[p-q, r(p)-r(q)] \leqq\|p-q\| \cdot\|r(p)-r(q)\|$, hence (20) implies that $r$ is nonexpansive.

Finally, if $E$ is smooth it follows from (8) and Lemma 1 that there can exist at most one orthogonal retraction of $C$ onto $F$.

EXAMPLE 1. In general, if $E$ is not smooth the nonexpansive projection need not be unique. Let $E=R^{2}$ normed by $\|(x, y)\|=$ $|x|+|y|$. If $r_{1}(x, y)=(0, y)$, then $r_{1}$ is a nonexpansive projection onto $L=\left\{(0, y): y \in R^{1}\right\}$. But $r_{2}(x, y)=(0, x+y)$ is also a nonexpansive projection of $E$ onto $L$, and $r_{1} \neq r_{2}$. 
If $E$ is not smooth, the implications (b) $\Rightarrow$ (a) and (c) $\Rightarrow$ (a) are false in general. Let $f: R^{1} \rightarrow R^{1}$ be any nonexpansive nonlinear function for which $f(0)=0$ (for example, $f=\arctan$ ). The retraction $r: E \rightarrow L$ defined by $r(x, y)=(0, f(x)+y)$ is easily calculated to be firmly nonexpansive, but it is not a projection.

THEOREM 2. Suppose $E$ is smooth, $F$ is a nonexpansive retract of $C$, and either

(a) $E$ is uniformly smooth, or

(b) $F$ is locally weakly compact and whenever $x_{n} \rightarrow 0$ weakly, $\left[y, x_{n}\right] \rightarrow 0$ for each $y \in E$.

Then there exists a nonexpansive projection of $C$ onto $F$.

Proof. By virtue of (8) and Theorem 1 it suffices to show that $F \cap A(p ; F) \neq \varnothing$ for each $p \in C$.

Let $f$ be any nonexpansive retraction of $C$ onto $F$, let $\lambda_{n} \rightarrow 1-$, and define $\left\{x_{n}\right\}$ by $x_{n}=\lambda_{n} f\left(x_{n}\right)+\left(1-\lambda_{n}\right) p$. By Lemma $3,\left\{x_{n}\right\}$ is bounded, and since

$$
x_{n}-f\left(x_{n}\right)=\left(1-\lambda_{n}^{-1}\right)\left(x_{n}-p\right)
$$

while $\lambda_{n} \rightarrow 1$, we have $x_{n}-f\left(x_{n}\right) \rightarrow 0$ strongly. But $f\left(x_{n}\right) \in F$, so $\lim _{n} \operatorname{dis}\left(x_{n}, F\right)=0$.

If hypothesis (a) holds, then by Lemma $2, F \cap A(p ; F) \neq \varnothing$ and we are finished.

If hypothesis (b) holds, set $y_{n}=f\left(x_{n}\right)$, so $y_{n} \in F$ and $x_{n}-y_{n} \rightarrow 0$. The sequence $\left\{y_{n}\right\}$ is therefore bounded, and since $F$ is locally weakly compact, there exists $y \in F$ and a subsequence of $\left\{y_{n}\right\}$ which converges weakly to $y$. Without loss of generality we may suppose $y_{n} \rightarrow y$ weakly. Then also $x_{n} \rightarrow y$ weakly. Since $x_{n} \in A(p ; F)$ and $y \in F$,

$$
\left[x_{n}-p, x_{n}-y\right] \leqq 0,
$$

which implies

$$
\left\|x_{n}-y\right\|^{2} \leqq\left[p-y, x_{n}-y\right]
$$

But $x_{n}-y \rightarrow 0$ weakly. By hypothesis (b), the right-hand-side (and hence the left-hand-side) of (21) goes to 0 . Thus $x_{n} \rightarrow y$ strongly.

Since $x_{n}-y_{n} \rightarrow 0$ strongly, $y$ is the strong limit of two sequences, $\left\{x_{n}\right\}$ in $A(p ; F)$ and $\left\{y_{n}\right\}$ in $F$. Since $F$ and $A(p ; F)$ are closed, $y \in$ $F \cap A(p ; F)$.

COROLlaRY 1. If $E$ is strictly convex and uniformly smooth, $T: C \rightarrow C$ is nonexpansive, and $F=\{x: T x=x\}$ is nonempty, then there exists a nonexpansive projection of $C$ onto $F$. 
Proof. $E$ is reflexive since $E^{*}$ is uniformly convex. By Theorem 1 of Bruck [4], $F$ is a nonexpansive retract of $C$; by Theorem 2(a) above, there exists a nonexpansive projection of $C$ onto $F$.

THEOREM 3. Suppose $F$ is a nonexpansive retract of $C$ which is locally compact, i.e., such that each bounded sequence in $F$ has a strongly convergent subsequence. Then there exists a firmly nonexpansive retraction of $C$ onto $F$.

Proof. Let $T$ be a nonexpansive retraction of $C$ onto $F$, and define the mappings $x_{\lambda}$ as in Lemma 3. By Lemma 3, T० $x_{\lambda}$ is seen to be a nonexpansive retraction of $C$ onto $F$. Set $f_{2}=T x_{2}$, and fix $y_{0} \in F$.

If we let $F_{p}=\left\{u \in F:\left\|u-y_{0}\right\| \leqq\left\|p-y_{0}\right\|\right\}$, then $F_{p}$ is compact and by Tychonoff's theorem the product $\prod_{p \in C} F_{p}$ is compact. Each $f_{\lambda}$. belongs to this product space since $f_{\lambda}$ is nonexpansive and $f_{\lambda}\left(y_{0}\right)=y_{0}$ :

$$
\left\|f_{\lambda}(p)-y_{0}\right\|=\left\|f_{\lambda}(p)-f_{\lambda}\left(y_{0}\right)\right\| \leqq\left\|p-y_{0}\right\| \text { • }
$$

If $0<t<1$ define $H_{t}$ to be the closure in $\Pi F_{p}$ of $\left\{f_{\lambda}: t \leqq \lambda<1\right\}$. The family $\left\{H_{t}: 0<t<1\right\}$ is seen to have the finite intersection property, and since each $H_{t}$ is compact, there exists $f$ in $\cap\left\{H_{t}: 0<t<1\right\}$. We claim that $f$ is firmly nonexpansive and retracts $C$ onto $F$.

If $\lambda_{n} \in(0,1)$ we abbreviate $f_{\lambda_{n}}$ and $x_{\lambda_{n}}$ to $f_{n}$ and $x_{n}$ respectively. It follows from the definition of the product topology and the fact that $f \in H_{t}$ for all $t \in(0,1)$ that for each $p, q \in C$ there exists a sequence $\lambda_{n} \rightarrow 1-$ such that $f_{n}(p) \rightarrow f(p)$ and $f_{n}(q) \rightarrow f(q)$.

But $x_{n}(p)-f_{n}(p) \rightarrow 0$ and $x_{n}(q)-f_{n}(q) \rightarrow 0$ since

$$
x_{n}-f_{n}=\left(1-\lambda_{n}^{-1}\right)\left(x_{n}-I\right) .
$$

Therefore, $x_{n}(p) \rightarrow f(p)$ and $x_{n}(q) \rightarrow f(q)$. Since $x_{n}$ is firmly nonexpansive (Lemma 3),

$$
\left\|(1-t)\left(x_{n}(p)-x_{n}(q)\right)+t(p-q)\right\| \geqq\left\|x_{n}(p)-x_{n}(q)\right\|
$$

for all $t \geqq 0$, so in the limit

$$
\|(1-t)(f p-f q)+t(p-q)\| \geqq\|f p-f q\| \cdot
$$

Therefore, $f$ is firmly nonexpansive.

That $f$ retracts $C$ onto $F$ is trivial. First, the range of $f$ is a subset of $F$ since $f \in \Pi F_{p}$. Next, if $p \in F$ we can choose $\lambda_{n} \rightarrow 1-$ such that $f_{n}(p) \rightarrow f(p)$. But $f_{n}(p) \equiv p$ since $p \in F$, therefore $f(p)=p$.

We have shown that $f$ is a firmly nonexpansive retraction of $C$ on $F$. 
4. Other results. Corollary 1 suggests the following question: can we find more general hypotheses than those of Corollary 1 which guarantee the existence of a nonexpansive projection onto the fixedpoint set of $T$ ? We can relax the nonexpansive condition if we strengthen the compactness assumption:

Theorem 4. Suppose $E$ is smooth and $T: C \rightarrow C$ is continuous and satisfies

$$
[T x-x, x-y] \leqq 0 \text { for all } x \in C, y \in F,
$$

where $F=\{x: T x=x\}$. If the image of $T$ is relatively compact then there exists a nonexpansive projection of $C$ onto $F$.

Proof. For $p \in C$ and $0<\lambda<1$, the map $\lambda T+(1-\lambda) p$ sends $C$ into $C$ and has a fixed point by Schauder's theorem. Let $x_{\lambda}$ denote such a fixed point. (Curiously, although there may exist many choices of $x_{\lambda}$ for a given $\lambda$, the strong $\lim _{\lambda \rightarrow 1-} x_{\lambda}$ exists independently of the choice of $x_{\lambda}$.)

Thus $\lambda\left(T x_{\lambda}-x_{\lambda}\right)=(1-\lambda)\left(x_{\lambda}-p\right)$. By Schauder's theorem, $F \neq \varnothing$. For any $y \in F$, we therefore have

$$
(1-\lambda)\left[x_{\lambda}-p, x_{\lambda}-y\right]=\lambda\left[T x_{\lambda}-x_{\lambda}, x_{\lambda}-y\right] \leqq 0
$$

by virtue of (22). Thus $x_{\lambda} \in A(p ; F)$.

Let $\lambda_{n} \rightarrow 1-$ and set $x_{n}=x_{\lambda_{n}}$. Since $T(C)$ is relatively compact, some subsequence of $\left\{T x_{n}\right\}$ converges strongly. Without loss of generality we may suppose $T x_{n} \rightarrow y$ in $C$. Since $x_{n}=\lambda_{n} T x_{n}+\left(1-\lambda_{n}\right) p$, it follows that also $x_{n} \rightarrow y$ and hence $T y=y$.

That is, $\left\{x_{n}\right\}$ is a sequence in $A(p ; F)$ which converges to a point $y \in F$. Since $A(p ; F)$ is closed, $y \in F \cap A(p ; F)$. Theorem 1 and (8) now imply the existence of a nonexpansive projection of $C$ onto $F$.

A concrete condition which implies (22) is that $T$ be quasi-nonexpansive, i.e., that whenever $x \in C$ and $T y=y$, there hold the inequality $\|T x-y\| \leqq\|x-y\|$. This is because

$$
[T x-y, x-y] \leqq\|T x-y\| \cdot\|x-y\| \leqq\|x-y\|^{2}=[x-y, x-y]
$$

from which (22) easily follows. We have proven:

Corollary 2. If $E$ is smooth and $T: C \rightarrow C$ is compact, continuous, and quasi-nonexpansive, then there exists a nonexpansive projection of $C$ onto the fixed-point set of $T$.

REMARK. It is not known whether smoothness of $E$ or compactness of $T$ are essential in this Corollary; except that any mapping 
without fixed points is vacuously quasi-nonexpansive, so that any such extension of the corollary would have to consider the empty set to be the range of a nonexpansive projection by convention.

Recently Karlovitz [9] has proven that if $\operatorname{dim} E=2$ then every closed convex subset of $E$ is the range of a nonexpansive projection. This follows easily from a result of [4] and our present results if we merely note that a line in $E$ is always a nonexpansive retract of $E$.

Theorem 5. If $\operatorname{dim} E=2$ and $F$ is a nonempty closed convex subset of $E$, then there exists a nonexpansive projection of $E$ onto $F$.

Proof. Let $L$ be a line in $E$. Then there exist $x_{0}, y_{0}$ with $\left\|x_{0}\right\|=1$ such that $L=\left\{y_{0}+t x_{0}: t \in R^{1}\right\}$. By a corollary of the Hahn-Banach theorem, there exists $w \in E^{*}$ with $\|w\|=1$ and $w\left(x_{0}\right)=1$. The mapping $f: E \rightarrow L$ defined by $f(x)=y_{0}+w\left(x-y_{0}\right) \cdot x_{0}$ is easily seen to be a nonexpansive projection of $E$ onto $L$. Thus every line in $E$ is the range of a nonexpansive projection.

It follows that any closed half-space $H$ in $E$ is a nonexpansive retract of $E$. Indeed, let $f$ be a nonexpansive projection of $E$ onto the boundary line $\partial H$ of $H$; define $g: E \rightarrow H$ by $g(x)=x$ for $x \in H$, $g(x)=f(x)$ for $x \notin H$. Obviously $g$ is a retraction of $E$ onto $H$. We claim that $g$ is nonexpansive.

We must prove that $\|g x-g y\| \leqq\|x-y\|$ for all $x, y \in E$. This is obvious if $x, y$ are both in $H$ or both in $E \backslash H$. Therefore, it is enough to show that $\|g(x)-y\| \leqq\|x-y\|$ whenever $x \notin H$ and $y \in H$. Let $z$ be the point of $\partial H$ which lies on the segment joining $y \in H$ to $x \in E \backslash H$. Then $g(x)-g(z)=f(x)-f(z)$ and $g(z)-y=z-y$, so

$$
\begin{aligned}
\|g(x)-y\| & \leqq\|g(x)-g(z)\|+\|g(z)-y\|=\|f(x)-f(z)\|+\|z-y\| \\
& \leqq\|x-z\|+\|z-y\|=\|x-y\|
\end{aligned}
$$

since $x, z, y$ are collinear. Therefore $g$ is nonexpansive and we have shown that $H$ is a nonexpansive retract of $E$.

Now suppose that $E$ is both smooth and strictly convex. (The general case will be proven later.) By Theorem 2 of Bruck [4], the intersection of nonexpansive retracts of a reflexive, strictly convex Banach space $E$ is also a nonexpansive retract of $E$. Since $F$ is the intersection of closed half-spaces, which are nonexpansive retracts of a two-dimensional space $E$, it follows that $F$ is a nonexpansive retract of $E$. A smooth finite-dimensional space is uniformly smooth, so by Theorem 2 (a) there exists a nonexpansive projection of $E$ onto $F$.

The case of a general norm $\|\cdot\|$ is treated as a limiting case. Let $\varepsilon_{n} \rightarrow 0+$ and for each positive integer $n$ choose a norm $\|\cdot\|_{n}$ on 
$E$ with respect to which $E$ is both smooth and strictly convex, and such that

$$
\|x\| \leqq\|x\|_{n} \leqq\left(1+\varepsilon_{n}\right)\|x\| \text {. }
$$

By our previous case, for each $n$ there exists a projection $f_{n}$ of $E$ onto $F$ such that $\left\|f_{n} x-f_{n} y\right\|_{n} \leqq\|x-y\|_{n}$ for each $x, y \in E$. Thus

$$
\left\|f_{n} x-f_{n} y\right\| \leqq\left\|f_{n} x-f_{n} y\right\|_{n} \leqq\left(1+\varepsilon_{n}\right)\|x-y\| \text {. }
$$

If $y_{0} \in F$ is fixed, we also have

$$
\left\|f_{n} x-y_{0}\right\|=\left\|f_{n} x-f_{n} y_{0}\right\| \leqq\left(1+\varepsilon_{n}\right)\left\|x-y_{0}\right\| \cdot
$$

Thus on bounded sets the family $\left\{f_{n}\right\}$ is equibounded and equicontinuous. By a variant of the Ascoli-Arzèla theorem (in which the mappings are into $E$, not $R^{1}$ ), there exists a subsequence of $\left\{f_{n}\right\}$ which converges uniformly on bounded sets to some $f: E \rightarrow E$. Without loss of generality we can suppose this subsequence is $\left\{f_{n}\right\}$ itself. It follows from (23) that $f$ is nonexpansive with respect to $\|\cdot\|$. Obviously $f x=x$ for all $x \in F$, while $f_{n} x \in F$ for all $n$ implies $f x \in F$. We have shown that $f$ is a nonexpansive retraction of $(E,\|\cdot\|)$ onto $F$.

To prove that $f$ is a projection, fix $t \geqq 0$ and $x \in E$ and set $x_{n}=$ $f_{n}(x), x_{n}^{*}=x_{n}+t\left(x-x_{n}\right)$, and $x^{*}=f(x)+t(x-f(x))$. Thus $x_{n} \rightarrow f(x)$, $x_{n}^{*} \rightarrow x^{*}$. But each $f_{n}$ is a projection, so

$$
f_{n}\left(x_{n}^{*}\right)=x_{n} \cdot
$$

The right-hand-side of (24) converges to $f(x)$, the left-hand-side to $f\left(x^{*}\right)$ since $f_{n} \rightarrow f$ uniformly on bounded sets and $x_{n}^{*} \rightarrow x^{*}$. Therefore, $f\left(x^{*}\right)=f(x)$, i.e., $f$ is a projection.

ExAmple 2. It may happen that there exists a nonexpansive projection of $C$ onto $F$ even if $F$ is not convex. For example, let $E=R^{2}$ normed by $\|(x, y)\|=\max (|x|,|y|)$, and define $f: E \rightarrow E$ by $f(x, y)=(x,|x|)$. Then $f$ is a nonexpansive projection of $E$ onto $F=$ $\{(x, y): y=|x|\}$, but $F$ is not convex.

\section{REFERENCES}

1. G. Birkhoff, Orthogonality in linear metric spaces, Duke Math. J., 1 (1935), 169-172. 2. F. E. Browder, Convergence of approximates to fixed points of nonexpansive nonlinear mappings in Banach spaces, Arch. for Rat. Mech. Anal., 24 (1967), 82-90.

3. - Convergence theorems for sequences of nonlinear operators in Banach spaces, Math. Zeitschr., 100 (1967), 201-225.

4. R. E. Bruck, Jr., Nonexpansive retracts of Banach spaces, Bull. Amer. Math. Soc., 76 (1970), 384-386.

5. D. G. de Figueiredo and L. A. Karlovitz, On the extension of contractions on normed spaces, Proc. Symp. in Pure Math. vol XVIII, Part I, Amer. Math. Soc., Providence, R. 
I., 95-104.

6. D. G. de Figueiredo and L. A. Karlovitz, On the radial projection in normed spaces, Bull. Amer. Math. Soc., 73 (1967), 364-368.

7. J. R. Giles, Classes of semi-inner-product spaces, Trans. Amer. Math. Soc., 129 (1967), 436-446.

8. R. C. James, Orthogonality and linear functionals in normed linear spaces, Trans. Amer. Math. Soc., 61 (1947), 265-292.

9. L. A. Karlovitz, The construction and application of contractive retractions in 2dimensional normed linear spaces, Tech. Note BN-717, Institute for Fluid Dynamics and Applied Mathematics, U. Maryland.

10. G. Lumer, Semi-inner-product spaces, Trans. Amer. Math. Soc., 100 (1961), 29-43. 11. V. L. Smulian, Sur la derivabilite de la norme dans l'espace de Banach, Dokl. Akad. Nauk SSSR (N. S.), 27 (1940), 643-648.

Received June 14, 1972. Partially supported by NSF Grant GP 30221.

University of SOUthern CaLifornia 



\section{PACIFIC JOURNAL OF MATHEMATICS}

\section{EDITORS}

D. Gilbarg AND J. MILGRAM

Stanford University

Stanford, California 94305

\section{R. A. Beaumont}

University of Washington

Seattle, Washington 98105

\section{J. DUGUNDJI*}

Department of Mathematics University of Southern California Los Angeles, California 90007

RICHARD ARENS

University of California Los Angeles, California 90024

\section{ASSOCIATE EDITORS}
E. F. BECKENBACH
B. H. NEUMANN
F. WOLF
K. YoshidA

\section{SUPPORTING INSTITUTIONS}

\author{
UNIVERSITY OF BRITISH COLUMBIA \\ CALIFORNIA INSTITUTE OF TECHNOLOGY \\ UNIVERSITY OF CALIFORNIA \\ MONTANA STATE UNIVERSITY \\ UNIVERSITY OF NEVADA \\ NEW MEXICO STATE UNIVERSITY \\ OREGON STATE UNIVERSITY \\ UNIVERSITY OF OREGON \\ OSAKA UNIVERSITY
}

\author{
UNIVERSITY OF SOUTHERN CALIFORNIA \\ STANFORD UNIVERSITY \\ UNIVERSITY OF TOKYO \\ UNIVERSITY OF UTAH \\ WASHINGTON STATE UNIVERSITY \\ UNIVERSITY OF WASHINGTON \\ AMERICAN MATHEMATICAL SOCIETY \\ NAVAL WEAPONS CENTER
}

The Supporting Institutions listed above contribute to the cost of publication of this Journal, but they are not owners or publishers and have no responsibility for its content or policies.

Mathematical papers intended for publication in the Pacific Journal of Mathematics should be in typed form or offset-reproduced, (not dittoed), double spaced with large margins. Underline Greek letters in red, German in green, and script in blue. The first paragraph or two must be capable of being used separately as a synopsis of the entire paper. Items of the bibliography should not be cited there unless absolutely necessary, in which case they must be identified by author and Journal, rather than by item number. Manuscripts, in duplicate if possible, may be sent to any one of the four editors. Please classify according to the scheme of Math. Rev. Index to Vol. 39. All other communications to the editors should be addressed to the managing editor, Richard Arens, University of California, Los Angeles, California, 90024.

50 reprints are provided free for each article; additional copies may be obtained at cost in multiples of 50 .

The Pacific Journal of Mathematics is issued monthly as of January 1966. Regular subscription rate: $\$ 48.00$ a year (6 Vols., 12 issues). Special rate: $\$ 24.00$ a year to individual members of supporting institutions.

Subscriptions, orders for back numbers, and changes of address should be sent to Pacific Journal of Mathematics, 103 Highland Boulevard, Berkeley, California, 94708.

PUBLISHED BY PACIFIC JOURNAL OF MATHEMATICS, A NON-PROFIT CORPORATION

Printed at Kokusai Bunken Insatsusha (International Academic Printing Co., Ltd.), 270, 3-chome Totsuka-cho, Shinjuku-ku, Tokyo 160, Japan.

* C. DePrima will replace J. Dugundji until August 1974.

Copyright (C) 1973 by

Pacific Journal of Mathematics

All Rights Reserved 


\section{Pacific Journal of Mathematics}

\section{Vol. 47, No. $2 \quad$ February, 1973}

David Parham Bellamy, Composants of Hausdorff indecomposable continua; a mapping approach ........................ 303

Colin Bennett, A Hausdorff-Young theorem for rearrangement-invariant spaces ...........................................

Roger Daniel Bleier and Paul F. Conrad, The lattice of closed ideals and $a^{*}$-extensions of an abelian l-group ...

Ronald Elroy Bruck, Jr., Nonexpansive projections on subsets of Banach

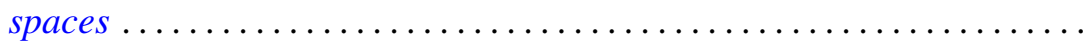

Robert C. Busby, Centralizers of twisted group algebras ............. 357

M. J. Canfell, Dimension theory in zero-set spaces ................ 393

John Dauns, One sided prime ideals ........................ 401

Charles F. Dunkl, Structure hypergroups for measure algebras . . . . . . . . . 413

Ronald Francis Gariepy, Geometric properties of Sobolev mappings ...... 427

Ralph Allen Gellar and Lavon Barry Page, A new look at some familiar spaces of intertwining operators ...........................

Dennis Michael Girard, The behavior of the norm of an automorphism of the

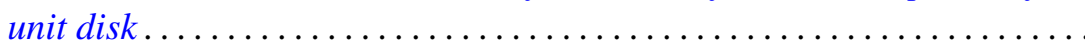

George Rudolph Gordh, Jr., Terminal subcontinua of hereditarily

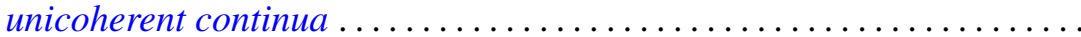

Joe Alston Guthrie, Mapping spaces and cs-networks. .

Neil Hindman, The product of $F$-spaces with $P$-spaces . 473

M. A. Labbé and John Wolfe, Isomorphic classes of the spaces $C_{\sigma}(S)$

Ernest A. Michael, On k-spaces, $k_{R}$-spaces and $k(X) \ldots$

Donald Steven Passman, Primitive group rings .

C. P. L. Rhodes, A note on primary decompositions of a pseudovaluation ...

Muril Lynn Robertson, A class of generalized functional differential equations

Ruth Silverman, Decomposition of plane convex sets. $I$.

Ernest Lester Stitzinger, On saturated formations of solvable Lie algebras................................

B. Andreas Troesch, Sloshing frequencies in a half-space by Kelvin inversion ...

L. E. Ward, Fixed point sets .

Michael John Westwater, Hilbert transforms, and a problem in scattering

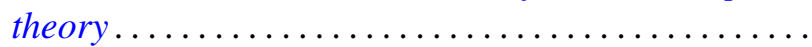

Misha Zafran, On the spectra of multipliers... 